\title{
Fibrinolytic Therapy for Thrombosis in Cardiac Valvular Prosthesis Short and Long Term Results
}

\author{
Auristela I. O. Ramos, Rui F. Ramos, Dorival J. D. Togna, Antoninho S. Arnoni, Rodolfo Staico, \\ Mercedes M. Galo, Zilda M. Meneghelo \\ São Paulo, SP - Brazil
}

\begin{abstract}
Objective - To assess the short- and long-term results of the use of streptokinase (SK) for the treatment of thromboses in cardiac valvular prostheses.

Methods - Seventeen patients with cardiac prosthetic thrombosis diagnosed by clinical, echocardiographic, and radioscopic findings underwent fibrinolytic treatment with a streptokinase bolus of 250,000 U followed by 100.000 U/hour. Short- and long-term results were assessed by radioscopy and echocardiography.
\end{abstract}

Results - Of the 17 patients, 12 had mechanical double-disk prostheses (4 aortic, 6 mitral, 2 tricuspid), 4 had single-disk prostheses (2 aortic, 1 mitral, and 1 tricuspid), and 1 had a tricuspid bioprosthesis. The success rate was $64.8 \%$, the partial success rate was $17.6 \%$, and the nonsuccess rate was $17.6 \%$. All patients with a double-disk prosthesis responded, completely or partially, to the treatment. None of the patients with a single-disk prosthesis had complete resolution of the thrombosis. The time of streptokinase infusion ranged from 6 to 80 hours (mean of $56 \mathrm{~h}$ ). The mortality rate due to the use of streptokinase was $5.8 \%$ and was secondary to cerebral bleeding. During streptokinase infusion, 3 (17.6\%) embolic episodes occurred as follows: 1 cerebral, 1 peripheral, and 1 coronary. The rethrombosis index was $33 \%$ in a mean follow-up of 42 months.

Conclusion - The use of fibrinolytic agents was effective and relatively safe in patients with primary thrombosis of a double-disk prosthesis. A fatal hemorrhagic complication occurred in 1 (5.8\%) patient, and embolic complications occurred in $3(17.6 \%)$ patients. In a mean 42month follow-up, $67 \%$ of the patients were free from rethrombosis.

Key words: fibrinolytic therapy, thrombosis, streptokinase, cardiac valvular prostheses

Instituto Dante Pazzanese de Cardiologia

Mailing address: Auristela Isabel de Oliveira Ramos - Av. Miruna, 327/44 Cep 04084-001 - São Paulo, SP, Brazil - E-mail: rafframos@uol.com.br

English version by Stela Maris C. e Gandour
Thrombosis in a cardiac valvular prosthesis is a rare, but severe complication. Its incidence ranges from $0.5 \%$ to $6 \%$ per patient per year in the aortic and mitral positions, depending on the degree of anticoagulation and the prosthetic design ${ }^{1}$. In 1971, Luluaga et $\mathrm{al}^{2}$ reported for the first time the successful use of fibrinolytic agents for the treatment of thrombosis in a Starr-Edwards prosthesis in the tricuspid position. From that time onwards, several cases have been reported ${ }^{3-6}$. The objective of this study was to analyze the use of the fibrinolytic agent, streptokinase, in the treatment of prosthetic thromboses.

\section{Methods}

This study prospectively analyzed patients with valvular prosthetic thrombosis diagnosed by clinical history and examination, transesophageal Doppler-echocardiography, and cinefluoroscopy.

The study comprised patients with diagnostic confirmation of prosthetic thrombosis, who were hemodynamically stable and considered of high surgical risk or with a contraindication for prosthesis replacement surgery. Patients with inadequate anticoagulation control, either due to lack of adherence to treatment, or lack of conditions to undergo periodical controls, and those with large thrombi in the atrial cavity were excluded from the study.

The diagnostic hypothesis of prosthetic thrombosis was raised when the patient reported worsening of his functional class, appearance of signs of low cardiac output, a history suggestive of transient embolism, or when the patient noticed a change in the intensity of the prosthetic click. The auscultatory findings of muffling of the metallic click, as well as the appearance or intensification of preexisting murmurs, contributed to the diagnostic suspicion. The fluoroscopy performed in the hemodynamics laboratory served as triage for all patients suspected of having prosthetic obstruction. Through fluoroscopy, the opening angle of the prosthesis, formed between the disk and the prosthetic ring, and the mobility of the disks were analyzed. The analysis of 
the disks requires an incidence in which the profile of the disks is filmed, and in which the disks are perpendicular to the prosthetic ring (figs. 1 and 2). Fluoroscopy was performed prior to beginning streptokinase infusion and was repeated every 24 hours to assess response to treatment.

Transesophageal Doppler-echocardiography complemented the clinical and fluoroscopic diagnosis. Echocardiography made possible the analysis of the mobility of the disks, the visualization of possible thrombi and their characteristics, such as size, location, and mobility, in addition to the calculation of transprosthetic gradients, valvular area, ventricular function, and the diameters of the cardiac cavities. The echocardiographies were performed before and after streptokinase infusion.

The diagnosis was established by associating the clinical findings and the echocardiographic and fluoroscopic findings.

Streptokinase was the fibrinolytic agent used in all patients. It was intravenously administered in a bolus of $250,000 \mathrm{U}$ for 30 minutes, followed by the infusion of 100,000 $\mathrm{U} /$ hour, in the coronary unit. Streptokinase infusion was suspended when the mobility of the prosthetic disks was normalized on fluoroscopy, in case of hemorrhagic complication, or when no improvement was observed in the mobility of the disks 72 hours after drug infusion. After suspension of the fibrinolytic agent, intravenous heparin was initiated, as soon as the partial thromboplastin time (PTT) was shorter than twice the control PTT. Oral anticoagulation with phenprocoumon was reinitiated 24 hours after the interruption of streptokinase in the patients who responded to treatment. They were discharged from the hospital receiving oral anticoagulation with the dose adjusted according to the International Normalized Ratio (INR) between 2.5 and 3.5 in association with $100 \mathrm{mg}$ of aspirin per day.

Late follow-up was performed on an outpatient care basis and with fluoroscopic and echocardiographic control every 6 months or when symptoms suggestive of thrombosis recurrence appeared.

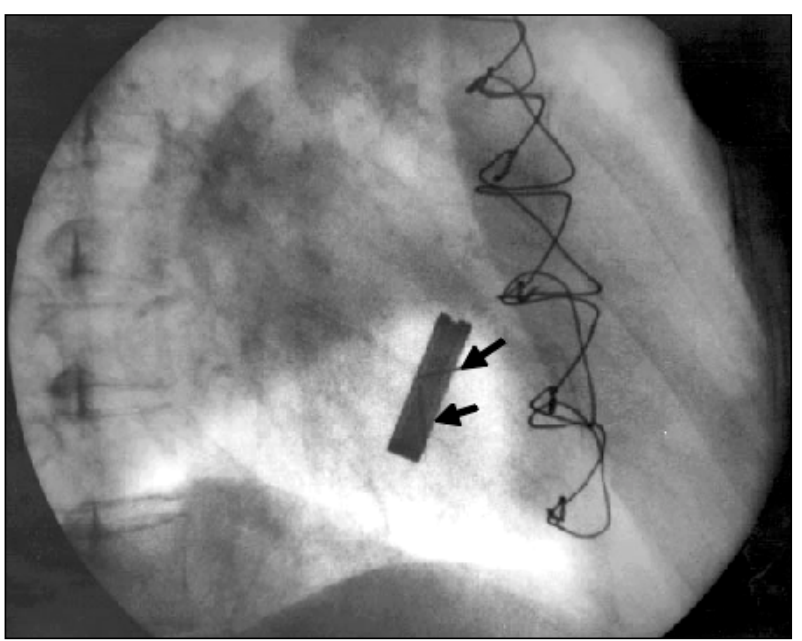

Fig. 1 - Radioscopy of the metallic double-disk prosthesis in the mitral position, prior to fibrinolysis. The upper arrow shows the small opening of the disk and the lower arrow shows the still disk.

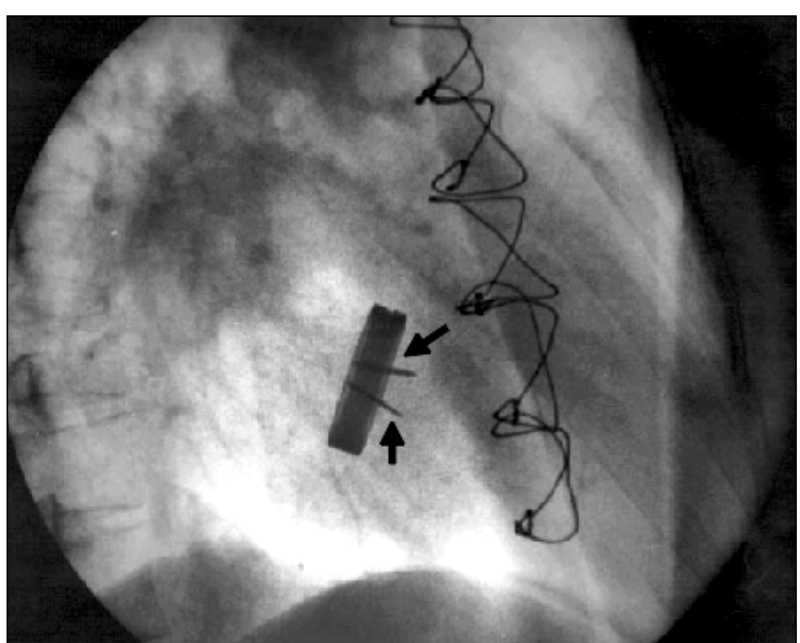

Fig.2 - Radioscopy of the metallic double-disk prosthesis in the mitral position, after fibrinolysis. Arrows show normal opening of the disks.

Successful treatment was defined as an improvement in clinical findings in association with normalization of the mobility of the disks and a reduction in the transprosthetic gradient. Partial success was defined as the improvement in clinical findings in association with a reduction in the transprosthetic gradient, but, on fluoroscopy, some reduction in the mobility of the disks still persisted. Nonsuccess was defined as the lack of resolution of the thrombosis, or when the patient had fatal complications.

\section{Results}

From January 1993 to July 2002, 17 inconsecutive patients, who met the inclusion criteria, received streptokinase for the treatment of thrombosis in the cardiac valvular prostheses. The mean age was 41 (12 to 60) years. Twelve were females. Twelve had mechanical double-disc prostheses (4 aortic, 6 mitral, 2 tricuspid), 4 had single-disk prostheses ( 2 aortic, 1 mitral, 1 tricuspid), and 1 had a porcine valvular prosthesis. Of these 12 patients, 8 had already undergone 2 or more previous valvular replacements. The INR was adequate, ie, between 2.5 and 3.5 , in only $6(35 \%)$ patients. Eleven (65\%) had inadequate anticoagulation with INR $<2$, due to the incorrect use of the medication or suspension of the anticoagulant agent for surgery. The patient with the porcine prosthesis was not receiving coumarin anticoagulants.

On physical examination, signs of congestive heart failure, appearance or intensification of murmurs of stenosis, and muffling of the metallic click were found in most patients.

The symptom most frequently reported by the patients was worsening of their physical capacity with the appearance of dyspnea associated with the perception of muffling of the metallic click of the prosthesis. One patient reported precordial pain with characteristics similar to those of angina, and another patient had an episode suggestive of transient cerebral ischemia with short-duration dyslalia. The duration of the symptoms related to a prosthetic thrombosis ranged from 3 to 90 days. 
The demographic characteristics, the model of the prosthesis, the time elapsed between prosthesis implantation and thrombosis, and the cardiac rhythm of patients with a prosthesis in the aortic and atrioventricular position are shown in tables I and II.

Of the 6 patients with an aortic prosthesis, 4 had double-disk prostheses and 2 had single-disk prostheses. Most patients had sinus rhythm. The time elapsed between prosthesis implantation and thrombosis ranged from 8 to 136 (mean of 72) months.

Of the 11 patients with atrioventricular prostheses (3 tricuspid and 8 mitral), 2 had single-disk prostheses, 8 had double-disk prostheses, and $1 \mathrm{had}$ a bioprosthesis. Three patients had atrial fibrillation, 6 had sinus rhythm, 1 had junctional rhythm, and 1 had pacemaker rhythm. The interval between the implantation of the valvular prosthesis and the diagnosis of thrombosis ranged from 1 to 120 (mean of 41) months. Two patients were still at the hospital after prosthesis implantation when thrombosis occurred. One of them had congenital heart disease, a very dilated right atrium with a tricuspid bioprosthesis complicated with respiratory infection and heart failure. The other patient had mitral and tricuspid metallic prostheses, which evolved with total atrioventricular block, which required heparin to replace phenprocoumon for definitive pacemaker implantation. Both patients were considered at high risk for reoperation.

In 11 of the 17 patients, the mobility of the disks was shown on fluoroscopy, coinciding with the drop in trans- prosthetic gradients, corresponding to a success rate of $64.8 \%$. Despite the drop in gradients, the disks maintained some degree of difficulty in opening in 3 patients, corresponding to a partial success rate of $17.6 \%$. The mean of the maximum aortic gradients dropped from $67 \mathrm{mmHg}$, prior to streptokinase, to $27 \mathrm{mmHg}$, after streptokinase; the mean of the mean atrioventricular gradients dropped from 14.5 $\mathrm{mmHg}$, prior to streptokinase, to $4.9 \mathrm{mmHg}$, after streptokinase. The nonsuccess rate was $17.6 \%$.

The duration of streptokinase infusion ranged from 6 to 80 (mean of 56) hours, being 34 hours in the patients who partially responded to the fibrinolytic agent, 44 hours in those who achieved no success, and 66 hours in those with resolution of the thrombosis (Tables III and IV).

Of the 4 patients with a single-disk prosthesis, only 1 (25\%) patient partially benefited from the fibrinolytic treatment. One patient died due to an intracranial hemorrhage, and the other 2 were referred for prosthesis replacement, but with no diagnostic confirmation by the surgeon, who reported the presence of a tissue formation suggestive of pannus surrounding the prosthetic ring.

Of the 12 patients with a double-disk prosthesis, complete resolution of the thrombosis occurred in $10(83 \%)$. Of the 2 patients with partial success, 1 was clinically managed, and the other was referred for surgical treatment, during which the prosthetic thrombosis was confirmed.

The mean time interval between prosthetic implantation and thrombosis was longer in the single-disk prostheses than in the double-disk prostheses (103 vs 33 months).

\begin{tabular}{|c|c|c|c|c|c|}
\hline $\mathrm{N}$ & Sex & Age (years) & $\begin{array}{l}\text { Type and size of the } \\
\text { thrombosed prosthesis }\end{array}$ & $\begin{array}{c}\text { Time between prosthesis implantation } \\
\text { and thrombosis (months) }\end{array}$ & Cardiac rhythm \\
\hline 1 & $\mathrm{~F}$ & 60 & ** CarboMedics Ao / 25 & 8 & Sinus \\
\hline 2 & M & 52 & ** CarboMedics Ao / 25 & 108 & Atrial flutter \\
\hline 3 & $\mathrm{~F}$ & 52 & * Omniscience Ao / 21 & 136 & Sinus \\
\hline 4 & M & 40 & * Omniscience Ao /25 & 72 & Sinus \\
\hline 5 & $\mathrm{~F}$ & 42 & ** St Jude Ao & 36 & Sinus \\
\hline 6 & M & 32 & ** St Jude Ao & 48 & Sinus \\
\hline
\end{tabular}

\begin{tabular}{|c|c|c|c|c|c|}
\hline $\mathrm{N}$ & Sex & Age (years) & $\begin{array}{l}\text { Type and size of the } \\
\text { thrombosed prosthesis }\end{array}$ & $\begin{array}{l}\text { Time between prosthesis implantation } \\
\text { and thrombosis (months) }\end{array}$ & Cardiac rhythm \\
\hline 1 & $\mathrm{~F}$ & 21 & $*$ M. Hall Tric / 25 & 84 & Juncional \\
\hline 2 & $\mathrm{~F}$ & 24 & ** TRI-technology Mi / 31 & 6 & Sinusal \\
\hline 3 & $\mathrm{~F}$ & 37 & ** CarboMedics Tric / 29 & 96 & Sinusal \\
\hline 4 & M & 38 & ** TRI-technology Mi / 31 & 7 & Fibril. Atrial \\
\hline 5 & $\mathrm{~F}$ & 53 & ** CarboMedics Mi / 31 & 38 & Sinusal \\
\hline 6 & $\mathrm{~F}$ & 59 & ** CarboMedics Mi / 31 & 1 & Fibril. Atrial \\
\hline 7 & $\mathrm{~F}$ & 12 & $* * *$ Labcor Tric / 29 & 2 & Sinusal \\
\hline 8 & M & 35 & * Omniscience $\mathrm{Mi} / 29$ & 120 & Fibril. Atrial \\
\hline 9 & $\mathrm{~F}$ & 52 & ** TRI-technology Tric / 29 & 1 & BAVT \\
\hline 10 & $\mathrm{~F}$ & 52 & ** CarboMedics Mi / 29 & 36 & Sinusal \\
\hline 11 & $\mathrm{~F}$ & 38 & $* *$ ATS Mi / 29 & 60 & Sinusal \\
\hline
\end{tabular}




\begin{tabular}{|c|c|c|c|c|c|c|}
\hline \multirow{3}{*}{$\mathrm{N}$} & \multicolumn{6}{|c|}{ Table III - Aortic prosthesis. Short and long-term results } \\
\hline & \multicolumn{2}{|c|}{$\begin{array}{l}\text { Maximum transprosthetic } \\
\text { gradient }(\mathrm{mmHg})\end{array}$} & \multirow[t]{2}{*}{$\begin{array}{l}\text { Duration of SK } \\
\quad \text { (hours) }\end{array}$} & \multirow[t]{2}{*}{ Results } & \multirow[t]{2}{*}{ Evolution } & \multirow[t]{2}{*}{$\begin{array}{c}\text { Follow-up } \\
\text { (months) }\end{array}$} \\
\hline & Pre-SK & Post-SK & & & & \\
\hline 1 & 68 & 24 & 24 & Partial & ** Rethrombosis & 39 \\
\hline 2 & 33 & 17 & 72 & Success & Normal & 51 \\
\hline 3 & 110 & 60 & 36 & * Nonsuccess & - & - \\
\hline 4 & 64 & 64 & 24 & Nonsuccess & Surgery & - \\
\hline 5 & 102 & 42 & 72 & Success & $* *$ Rethrombosis & 11 \\
\hline 6 & 80 & 30 & 6 & Success & Normal & 6 \\
\hline
\end{tabular}

\begin{tabular}{|c|c|c|c|c|c|c|}
\hline \multicolumn{7}{|c|}{ Table IV - Mitral and tricuspid prosthesis. Short and long-term results } \\
\hline \multirow[t]{2}{*}{$\mathrm{N}$} & \multicolumn{2}{|c|}{$\begin{array}{l}\text { Mean transprosthetic } \\
\text { gradient }(\mathrm{mmHg})\end{array}$} & \multirow[t]{2}{*}{$\begin{array}{l}\text { Duraction of } \\
\text { SK (hours) }\end{array}$} & \multirow[t]{2}{*}{ Results } & \multirow[t]{2}{*}{ Evolution } & \multirow[t]{2}{*}{$\begin{array}{c}\text { Follow-up } \\
\text { (months) }\end{array}$} \\
\hline & Pre-SK & Post-SK & & & & \\
\hline 1 & 09 & 05 & 06 & Partial & $* *$ Rethrombosis & 36 \\
\hline 2 & 13 & 07 & 72 & Success & Normal & 60 \\
\hline 3 & 07 & 03 & 72 & Success & Normal & 48 \\
\hline 4 & 11 & 05 & 20 & Success & Normal & 54 \\
\hline 5 & 10 & 05 & 72 & Success & Normal & 48 \\
\hline 6 & 07 & 03 & 60 & Success & No evolution & - \\
\hline 7 & 14 & 06 & 72 & Success & Normal & 36 \\
\hline 8 & 23 & 23 & 72 & Nonsuccess & Surgery & - \\
\hline 9 & 17 & 4 & 72 & Success & Normal & 22 \\
\hline 10 & 29 & 5 & 80 & Success & Rethrombosis & 15 \\
\hline 11 & 28 & 6 & 72 & Partial & Surgery & - \\
\hline
\end{tabular}

The most common hemorrhagic complication was hematoma in the venous puncture site. One (5.8\%) fatal cranial bleed occurred, as already cited, in a patient with a single-disk prosthesis in the 36th hour of streptokinase infusion.

Three (17.6\%) embolic phenomena occurred. One patient had peripheral embolism to the left radial artery, which resolved with maintenance of streptokinase. A second patient had an ischemic stroke; streptokinase infusion was interrupted, bleeding was ruled out on cranial tomography, and, after 2 weeks, the patient was referred for surgery. The third patient had intense precordial pain with elevation of the ST segment in the anterior wall; he was referred to the hemodynamics laboratory. Embolism to the anterior descending artery was diagnosed and treated with angioplasty and stent implantation. Two hours later, the patient had a new episode of pain and elevation of the ST segment in the inferior wall, returning to the hemodynamics laboratory, where embolism to the right coronary artery was diagnosed and treated with angioplasty and stent implantation.

Of the 13 patients being discharged from the hospital, 1 did not return for follow-up and the other 12 underwent a mean follow-up of 42 months. Four patients (33\%) had clinical, fluoroscopic, and echocardiographic rethrombosis within a mean interval of 16 months. Of these 4 patients, 2 had single-disk prostheses with residual difficulty in disk mobility; the other 2 had double-disk prostheses and successfully responded to the initial treatment. One patient with a mitral double-disk prosthesis refused to undergo the surgical treatment, and as the patient had already received streptokinase within the 12 preceding months, the following medication scheme was prescribed: $10,000 \mathrm{U}$ of subcutaneous heparin, twice a day, $200 \mathrm{mg}$ of aspirin, and an oral anticoagulant agent for 3 months. The patient evolved with normalization of the mobility of the disks and a drop in the transprosthetic gradients, which remained unaltered after 7 months. For the other 3 patients, a new fibrinolytic treatment was indicated: 1 patient with an aortic double-disk prosthesis successfully responded, but, 12 months later, a new thrombosis occurred in the prosthesis, and the patient was referred for valvular replacement, during which the diagnosis was confirmed. The other patient with an aortic double-disk prosthesis had an episode of cerebral embolism, and, after stabilization, was referred for surgery, during which thrombosis was confirmed. The patient with a tricuspid single-disk prosthesis successfully responded to streptokinase infusion, and, on the control performed after 17 months of follow-up, the prosthesis was functioning normally. The patient with the bioprosthesis is asymptomatic and the transprosthetic gradient on echocardiography was low on the 36-month follow-up.

\section{Discussion}

The diagnosis of prosthetic thrombosis should always be remembered in patients with clinical findings of embolism 
or heart failure of recent onset, and symptoms of low cardiac output, mainly in patients with inadequate anticoagulation.

The isolated calculation of transprosthetic gradients is not sufficient to establish the diagnosis of prosthetic thrombosis, because these gradients may be elevated due to changes in flow, especially in the single-disk prostheses and those with small diameters. Therefore, a serial echocardiographic assessment is fundamental for the patients, to allow a longitudinal comparison of the gradients and the differential diagnosis between acute dysfunction due to thrombosis and that due to prosthesis-patient mismatch. In these cases, the clinical scenario is not acute, the prosthesis being usually implanted in the patient while still young. However, the diagnosis of thrombosis may be confirmed if images of the thrombus or anomalies in the mobility of the disks are visualized on transesophageal echocardiography or fluoroscopy. Fluoroscopy has helped in diagnosing prosthetic obstruction due to thrombosis, mainly in double-disk prostheses, in which the 2 disks may be compared in regard to mobility and opening angles.

The distinction between pannus and thrombus is complex, because both may present as masses and impede opening and closing of 1 or 2 disks. The old prostheses and the chronicity of the clinical findings were the major clinical characteristics found in a series of 12 patients with pannus reported by Sanchez et al $^{7}$.

Thrombosis of the prosthesis is a permanent risk for cardiac valvular prostheses, despite the anticoagulant therapy and improvement in their performance. The surgery for prosthesis replacement has been the traditional treatment, but the surgical risk ranges from 0 to $69 \%$, according to some series $^{8,11,12}$, causing the search for other therapeutic possibilities.

Fibrinolytic therapy has been described as an alternative to surgical treatment, being considered the first-line treatment in prosthetic thrombosis in the tricuspid position ${ }^{8,9}$. The use of a fibrinolytic agent for treating thrombosis in valvular prostheses in the mitral and aortic positions remains controversial. Some authors have indicated fibrinolytic therapy only for critically ill patients, in NYHA functional class III or IV, in whom the surgical intervention is of high risk, or in patients with contraindications ${ }^{8-12}$. The controversy in regard to the use of fibrinolytic therapy in patients in functional class I or II is based on the low surgical risk observed in this group of patients as compared with the thromboembolic risk caused by fibrinolysis, which ranges from 12 to $17 \%{ }^{10-13}$. On the other hand, some authors have indicated fibrinolysis as the first line of therapy in patients with St. Jude prostheses with a low risk of permanent complications and an excellent chance of success ${ }^{3}$. Among us, Campagnucci et al ${ }^{5}$ reported the results of fibrinolytic therapy for treating prosthetic thrombosis in 8 patients with a success rate of $100 \%$ and fatal complication rate of zero. More recently, Baptista Filho et al ${ }^{6}$ reported the successful use of streptokinase in a 75-year-old female patient with thrombosis in a metallic prosthesis in the mitral position on the 45 th postoperative day of myocardial revascularization.

In our study, we selected the stable patients or those with elevated surgical risk due to antecedents of 1 or more previous surgeries, or patients, who, due to some reason, had their anticoagulation temporarily suspended or poorly controlled, ie, who had a transient reason for having a prosthetic thrombosis.

The subcutaneous use of heparin in association with an anticoagulant and an antiplatelet agent has also been indicated for patients with partial thrombosis of the prosthesis who are mildly symptomatic ${ }^{8}$. In this series, 1 patient with rethrombosis successfully responded to that therapeutic strategy.

One patient died and the mortality rate $(5.8 \%)$ was acceptable considering the severity of prosthetic thrombosis. The risk of embolism was greater than that of bleeding $(15 \%$ vs 5.8\%). Peripheral embolism was resolved with maintenance of the fibrinolytic treatment in agreement with the literature ${ }^{14}$. The patient who had a stroke had his streptokinase suspended, underwent cranial tomography to rule out bleeding, and was referred for surgery 2 weeks later. The third patient complicated with 2 coronary embolisms, which were treated with a percutaneous intervention. A hemorrhagic complication was fatal in 1 patient.

Although the immediate postoperative period may be considered a relative contraindication to fibrinolytic therapy, 2 patients with prostheses in the tricuspid position received streptokinase while at the hospital, approximately 30 days after valvular replacement, and developed no hemorrhagic complications ${ }^{8,15}$.

In patients with single-disk prostheses, the success rate was zero, the nonsuccess rate was $75 \%$, and partial success was obtained only in 1 patient, who evolved with rethrombosis of the prosthesis in the 19th month of evolution. This may have been due to the advanced age of the prostheses (mean time of implantation of 103 months) and to their own design. These factors propitiate the formation of pannus and allow the fibrinolytic therapy to partially solve the prosthetic obstruction or not alter it at all.

On the other hand, the nonsuccess rate was zero in the double-disk prostheses with a shorter interval of implantation, thrombosis being the primary cause of obstruction.

Based on the mean time of streptokinase infusion, the nonsuccess rate was also observed to be greater in those receiving streptokinase for a shorter period.

Despite the small number of patients, our conclusions were as follows: the diagnosis of thrombosis of single-disk prostheses is hindered by the formation of pannus, which is frequent in old prostheses; thromboembolic complications were more common than hemorrhagic ones; and the patients with partial success evolved to rethrombosis. The treatment with streptokinase is an alternative to surgical treatment in select cases, such as those of thrombosis in a double-disk prosthesis. 


\section{References}

1. Edmunds LH. Thromboembolic complications of current cardiac valvular prostheses. Ann Thorac Surg 1982;34:96-106.

2. Luluaga IT, Carrera D, D'Oliveira J, et al. Successful thrombolytic therapy after acute tricuspide valve obstruction. Lancet 1971;1:1067-8.

3. Silber H, Khan SS, Matloff JM, Chaux A, DeRobertis M, Gray R. The St. Jude valve: thrombolysis as the first line of therapy for cardiac valve thrombosis. Circulation 1993;87:30-7.

4. Vasan RS, Kaul U, Sanghvi S, et al. Thrombolytic therapy for prosthetic valve: a study based on serial Doppler-echocardiographic evaluation. Am Heart J 1992; $123: 1575-80$

5. Campagnucci VP, Sukuzi HY, Franken RA, Rivetti LA. Terapêutica trombolítica nas tromboses de próteses mecânicas. Arq Bras Cardiol 1994, 63:35-8.

6. Baptista Filho MLA, SucciJE, Galantier M, et al. Terapêutica trombolítica em trombose de prótese mitral e átrio esquerdo. Rev Bras Cardiol Invas 2001;9:34-8.

7. Sänchez Ramos JG, DiazJ, Moreno G, NavarreteA, Prades I, Martin P. Predictores clínicos de trombosis em prótesis valvulares mecânicas. Rev Soc Andal Card $1998 ; 16: 45$
8. Lengyel M, Fuster V, Keltai M, et al. Guidelines for management of left-sided prosthetic valve thrombosis: a role for thrombolytic therapy. J Am Coll Cardiol 1997;15:1521-6

9. Peterfly A, HenkeA, Savidge GF, Landon C, Bjork VO. Late thrombotic malfunction of the Bjork-Shiley tilting disc valve in the tricuspide position. Scand $\mathrm{J}$ Thorac Cardiovasc Surg 1980;14:33-8.

11. Birdi I, Angelini GD, Bryan AJ. Thrombolytic therapy for left-side prosthetic valve thrombosis. J Heart Valve Dis 1995;4:154-9.

12. Roudat R, Labbe T, Lorient Roudaut MF, et al. Mechanical cardiac valve thrombosis: is fibrinolysis justified? Circulation 1992:86 (suppl II) II-8-15

13. Witchitz S, Veyrat C, Moisson P, Scheinman N, Rozenstajn L. Fibrinolytic treatment of thrombus on prosthetic heart valves. Br Heart J 1980;44:545-54.

14. Kurzrock S, Singh AK, Most AS, Williams DO. Thrombolytic therapy for prosthetic cardiac valve thrombosis. J Am Coll Cardiol 1987;9:592-8.

15. Asante Korang A, Sreeram N, McKay R, Arnold R. Thrombolysis with tissue type plasminogen activator following cardiac surgery in children. Int J Cardiol 1992; $35: 317-22$



Editor da Seção de Fotografias Artísticas: Cícero Piva de Albuquerque

Correspondência: InCor - Av. Dr. Enéas C. Aguiar, 44 - 05403-000 - São Paulo, SP - E-mail: delcicero@incor. usp.br 